DOI: https://doi.org/10.46296/yc.v5i9edespsoct.0108

\title{
TÉCNICAS CREATIVAS DE COMPRENSIÓN LECTORA Y COMPETENCIAS LINGÜÍSTICAS EN LA BÁSICA MEDIA
}

\section{CREATIVE READING COMPREHENSION TECHNIQUES AND LINGUISTIC COMPETENCIES IN MIDDLE SCHOOL}

\author{
Rodríguez-Salmerón Sandra Mariuxi ${ }^{1}$; Vélez-Villavicencio Carlina Edith ${ }^{2}$ \\ ${ }^{1}$ Universidad San Gregorio de Portoviejo USGP. Portoviejo Ecuador. Correo: \\ sandrars1985@hotmal.es. ORCID ID: https://orcid.org/0000-0003-1060-4433 \\ 2 Universidad San Gregorio de Portoviejo USGP. Portoviejo Ecuador. Correo: \\ cevelez@sangregorio.edu.ec. ORCID ID: https://orcid.org/0000-0003-3301-142X
}

\section{Resumen}

Esta investigación estudia las técnicas creativas de comprensión lectora y las competencias lingüísticas en el aprendizaje de los discentes. El objetivo de esta investigación fue indagar sobre las técnicas creativas de comprensión lectora factibles en el proceso de enseñanza virtual. Esta investigación fue no experimental, se trabajó en forma sistemática y se profundizo en el estudio de las variables. En cuanto al estudio exploratorio se procedió a investigar los alcances descriptivos, correlacionales o explicativos que fue la base de la investigación en la comprensión lectora de las competencias lingüísticas. Para valorar las competencias lingüísticas se planteó la aplicación de técnicas creativas con estudiantes considerado como grupo focal a través de una batería de preguntas que permitió valorar el alcance de las competencias lingüísticas. Se aplicó encuesta a docentes y entrevista a experto para el análisis de estudio. El estudio demostró que 8 de cada 10 niños no comprende lo que leen, por lo tanto, el docente debe aplicar nuevas técnicas, trabajar contenidos distintos y contar con materiales de lectura que estén vinculados a su entorno. Se requiere proponer la aplicación de técnicas creativas de comprensión lectora para mejorar la competencia lingüística de los estudiantes, por tal razón los docentes deben continuar capacitándose y formándose por medio de jornadas pedagógicas en las que se reflexione en lo que respecta a las dificultades en la comprensión lectora para buscar alternativas y soluciones a estas falencias.

Palabras claves: Comprensión lectora, competencias lingüísticas, técnicas creativas.

\begin{abstract}
This research studies creative reading comprehension techniques and linguistic competences in students' learning. The objective of this research was to investigate the creative reading comprehension techniques feasible in the virtual teaching process. This research was nonexperimental, worked systematically and studied the variables in depth. As for the exploratory study, we proceeded to investigate the descriptive, correlational or explanatory scopes, which was the basis of the research on reading comprehension of linguistic competences. In order to assess the linguistic competences, the application of creative techniques with students was considered as a focal group through a battery of questions that allowed assessing the scope of linguistic competences. A survey was applied to teachers and an interview with an expert for the study analysis. The study showed that 8 out of 10 children do not understand what they read; therefore, teachers should apply new techniques, work with different contents and have reading materials that are linked to their environment. It is necessary to propose the application of creative reading comprehension techniques to improve the linguistic competence of the students. For this reason, teachers should continue their training and education through pedagogical workshops in which they reflect on the difficulties in reading comprehension in order to find alternatives and solutions to these shortcomings.
\end{abstract}

Keywords: Reading comprehension, language skills, creative techniques.

Información del manuscrito:

Fecha de recepción: 22 de julio de 2021.

Fecha de aceptación: 24 de septiembre de 2021.

Fecha de publicación: 01 de octubre de 2021. 


\section{Introducción}

La investigación abordó el problema referente al insuficiente uso de técnicas creativas en la comprensión lectora que incides en las competencias lingüísticas en los estudiantes de la básica media.

Lo expuesto por (Salazar, 2018, pág. 1) a nivel mundial indica que los estudiantes no se interesan por leer, debido a ello, se ve seriamente comprometida la comprensión lectora, con esta afirmación se enfatiza que los docentes deben poner mucho énfasis en la enseñanza aprendizaje en lo que respecta a la comprensión lectora, que sin duda impacta en el accionar educativo.

Otra problemática expuesta por (Miranda, 2015, pág. 7) menciona que los profesores con frecuencia se preguntan ¿Cómo enseñar a los alumnos a entender, o a comprender lo que leen? Es necesario encontrar, una iniciativa en estar en constate renovación con técnicas de comprensión lectora, a fin, de que los estudiantes mejoren su aprendizaje con nuevas técnicas de enseñanza apoyadas en la comprensión de la lectura.
Lo expuesto por (Pacheco, 2018, pág. 7) en el Ecuador aún falta que se lleve a cabo realmente la implementación de estrategias didácticas que faciliten el aprendizaje de las diversas competencias lingüísticas. Se mencionan algunas; ausencia de capacitaciones al docente, carencia de recursos y materiales o tiempo limitado.

Por tales razones el objetivo de este artículo radica en indagar las técnicas creativas de comprensión lectora factibles en el proceso de enseñanza virtual. Teniendo en cuenta que estas técnicas sean empleadas correctamente por el docente para el debido aprendizaje en general, estas técnicas desarrollarán conocimientos y habilidades a través del mismo permitiendo que el estudiante las aplique, en el periodo de escolarización, por ello la importancia de su desarrollo, ya que este favorecerá de manera directa a los escolares que están en pleno desarrollo lector y educativo.

Para las autoras, técnicas creativas de comprensión lectora, significa los procesos de enseñanza y aprendizaje como herramientas 
psicopedagógicas de mayor importancia, debido al carácter cognitivo, afectivo y conductual que esta conlleva, así como una habilidad cognitiva necesaria para que los estudiantes logren un mejor desempeño en la realización de sus asignaciones. En consonancia al autor (Cervantes, 2017, pág. 4) indica que comprensión lectora es parte fundamental en la vida de todo estudiante y docente, ya que, a través de ella, se puede emprender la lectura de textos con el objetivo de comprender el significado del escrito, para posteriormente incrementar el bagaje de conocimiento del lector.

A continuación, se aborda el estudio de técnicas creativas para la comprensión lectora:

\section{Transformación de la narración.}

Luego de que los estudiantes han leído uno de los textos sugeridos por el profesor, deben construir una narración oral con elementos de apoyo para su presentación pública. Lo esencial es que los estudiantes demuestren que han leído y comprendido el texto y representen su propia interpretación del mismo. (Chavez, 2015). Esta estrategia didáctica puede ser grupal, provocando que los estudiantes usen formas opcionales para implantar sus conocimientos, siendo un suplente para las evaluaciones escritas que eran manejadas para saber que tanto se había comprendido de una lectura establecida.

\section{Las tramas narrativas}

Después de haber leído una narración, el estudiante tratará de recordar el texto que ha construido y almacenado en la memoria y lo escribirá en un armazón que puede haber sido confeccionado por el profesor 0 por los propios estudiantes. Posteriormente se deberá estimular a los estudiantes para que comparen si la trama que han escrito coincide con la del relato original. (Chavez, 2015). Esta estrategia favorece a los leyentes a elaborar y comparar una comprensión racional del texto anteriormente leído.

\section{Fichas de personajes}

Se puede pedir a los estudiantes que dibujen en una cartulina la silueta de un personaje y después que hagan una lista dentro de la silueta con las diferentes características de los 
mismos. Éstas se pueden exponer y pegar en el pizarrón debajo del título de la historia a la que corresponda. De esta manera se puede visualizar las distintas relaciones que se dan entre los personajes. (Chavez, 2015). Esta estrategia fue planteada con el fin de reunir la curiosidad y la atención de los estudiantes en las cualidades de los actores de un texto específico. Haciendo que el docente observe la compresión que ha tenido el educando de los diferentes personajes con sus hechos.

\section{Conversación escrita con un personaje}

En esta estrategia, el alumno elabora un diálogo escrito con un personaje de un texto leído. El objetivo de esto es estimular a los estudiantes a que se centren en un personaje específico y traten de comprender su forma de pensar, hablar y escribir. (Chavez, 2015). Como se dijo anteriormente, es una estrategia que los docentes acogen para saber si los estudiantes han comprendido el trabajo que realizó cada personaje que se encuentre en un texto indicado.

\section{Anuncio de una historia}

Comentar con los estudiantes varios detalles y características de una historia o libro que hayan leído recientemente todo el grupo de clase. Luego, en grupos, sugiéreles que elaboren un anuncio con las características más importantes de la obra, de tal manera que puedan promocionar su lectura a otros alumnos y amigos. No olvidar explicar los principios de un buen anuncio, de igual manera incítalos a realizar anuncios de otras obras y a coleccionarlos. (Chavez, 2015). Esta estrategia será diseñada con el fin de saber si la lectura que se realizó en clases fue comprendida y atrayente al estudiante de tal manera que realice un anuncio con características fundamentales $y$ esenciales de la lectura fomentando un interés necesario hacia sus demás compañeros.

Para finalizar, estas estrategias didácticas serán un buen manejo para que los docentes puedan provocar más interés hacia los estudiantes, fomentando el beneficio de la lectura en ellos. 
Factores que intervienen en la comprensión lectora.

En el proceso de la comprensión lectora se presentan factores importantes que deben considerar los docentes como parte del desarrollo de la compresión lectora: la memoria, el vocabulario, las técnicas y los estímulos a los estudiantes.

La memoria para (Laguardia, 2016) resultó ser un buen predictor de los problemas de comprensión lectora. Al parecer la memoria de trabajo, específicamente su componente verbal, constituye un predictor significativo de las habilidades de decodificación y comprensión lectora, constituye la única de las funciones ejecutivas que predice significativamente la competencia lectora en años posteriores.

Por tal motivo la memoria del estudiante debe ser desarrollada y tomada en cuenta como el papel fundamental por los docentes, porque estarán reflejadas todas las experiencias pasadas y presentes durante su vida escolar y su aprendizaje.
Para (Laguardia, 2016), el vocabulario es uno de los mejores predictores para un desempeño adecuado en comprensión lectora, pues no conoce el uso o sentido de determinadas palabras se convierte en un obstáculo importante para el logro de una representación integrada que dé cuenta de la comprensión

Otro de los factores indispensables mencionados por (Moren, 2018) es el estímulo del alumno se hace factible que lo docentes varíen constantemente su metodología de enseñanza. Hacer de la asistencia a su clase una experiencia enriquecedora, sorpresiva y variada, ya que la rutina puede ser el comienzo de la desmotivación, existen multitud de recursos didácticos que puede utilizar los docentes para estimular a los estudiantes y mantener el interés hacia el aprendizaje en sus clases.

Es preciso indicar que en este estudio se consideran competencias lingüísticas como la suma de conocimientos, destrezas y características individuales que permiten a una persona realizar acciones. Estas competencias lingüísticas deben manifestarse a la 
hora de comprender un texto, expresar un mensaje o simplemente con el fin de realizar una tarea según el autor (Cordero Badilla, 2018, pág. 277).

Tomando en consideración que de acuerdo al currículo educativo de la básica media (Ministerio de Educación del Ecuador, 2016, pág. 45) indica que: "El rol de la escuela en el área de Lengua y Literatura es ampliar, incrementar, acompañar y enriquecer todas las capacidades lingüísticas, estéticas y de pensamiento de los estudiantes, durante su proceso formativo".

Estudiando algunos autores, destacan la importancia de la comprensión lectora para el desarrollo progresivo e indispensable del hecho lector, como son las competencias de leer, escribir, escuchar y hablar.

\section{Competencia de leer}

Para (Ortega, 2017) los estudiantes no están motivados para leer, nunca alcanzarán su potencial de alfabetización completo. Se pretende fomentar la motivación intrínseca del estudiantado, es decir, su motivación de leer para disfrutar, de este modo, alcanzar el disfrute, se promoverá que los educandos afronten la lectura con mejores expectativas $y$ tengan en mayor consideración su autoeficacia lectora para el desarrollo de esta competencia, para ampliar el conocimiento y formar estudiantes con capacidades de reflexión y opinión sobre lo que leen.

\section{Competencia de Escuchar}

Menciona (Neyra, 2017) que el escuchar es considerado como uno de los componentes más importantes del aprendizaje de idiomas, no puede ser visto de manera estática y unidireccional, sino más bien como un proceso de comprensión auditiva consistente en una serie de pasos que inicia con la recepción de ondas de sonido a través del oído y la transmisión de impulsos nerviosos al cerebro, que luego activará impulsos que dan lugar a un gran número de mecanismos afectivos y cognitivos

Es necesario que la escuela desarrolle en los estudiantes todas las formas de escucha mediante situaciones de interacción comunicativa en las cuales se produzca un intercambio efectivo de 
papeles entre emisor y receptor (cuando se escucha para intervenir, la intención es más intensa en la medida que está motivada y dirigida) y en las que se prepare al alumno para la verdadera comprensión oral.

\section{Competencia Hablar}

Para (Neyra, 2017) hablar está estrechamente relacionada con la habilidad de escuchar, desarrollándose ambas simultáneamente. La forma más habitual de alcanzar niveles más elevados de dominio del idioma en estas habilidades es a través de la conversación.

Es menester recordar que todo buen profesor sabe que el éxito o fracaso de una intervención oral radica fundamentalmente en el mayor o menor esfuerzo y tiempo invertido en su preparación. Los profesores que no planean sus clases difícilmente obtendrán resultados satisfactorios. A falta de preparación, se emplea la improvisación con consecuencias lamentables: los estudiantes lo notan de inmediato y responden con desinterés y falta de disciplina.

\section{Competencia Escribir}

Según (Neyra, 2017) el concepto de escritura se vincula a la acción y las consecuencias del verbo escribir, consistente en plasmar pensamientos en un soporte material, para lo que se hará uso de signos lingüísticos específicos. Estos signos, por lo general, son letras que forman palabras, las que a su vez se unen para formar oraciones y así transmitir un mensaje determinado.

Estas competencias lingüísticas son las que los estudiantes deben dominar para comunicarse eficazmente en todas las situaciones posibles. No se puede utilizar el lenguaje con finalidades comunicativas si no se dominan estas habilidades lingüísticas, también llamadas destrezas y macro habilidades.

Para el trabajo dinámico de estas cuatro competencias, es necesario que el educador tenga claro cuáles son las competencias específicas por trabajar de manera intensiva y gradual en cada uno de los niveles educativos, con el propósito de contribuir efectivamente con el desarrollo de la aptitud comunicativa 
de los educandos, mediante la puesta en práctica de secuencias didácticas para incrementar la comprensión lectora.

\section{Metodología (Materiales y métodos)}

La actual investigación tuvo un enfoque cuali-cuantitativa ambos tipos fueron de gran utilidad para identificar las técnicas creativas de comprensión lectora y las competencias lingüísticas que sirvieron de base a la investigación. Se utilizó una investigación bibliográfica, mediante estudios actualizados y especializados del tema a investigar.

El tipo de investigación fue no experimental esto nos permitió observar situaciones ya existentes en las técnicas creativas de la compresión lectora en las competencias lingüísticas, se trabajó en forma sistemática y se profundizo en el estudio de las variables.

En cuanto al estudio exploratorio se preparó el terreno para proceder a investigar los alcances descriptivos, correlacionales 0 explicativos. El estudio descriptivo ayudó como base de la investigación en la comprensión lectora de las competencias lingüísticas, las cuales proporcionaron información mediante el estudio explicativo.

La investigación responde al método Analítico - Sintético, que sirvió para seleccionar el objeto de estudio analizar cada una de sus partes y luego sintetizar nuevamente la información; Inductivo - deductivo servirá para construir una investigación sólida y fundamentada correctamente a nivel teórico.

En cuanto a técnicas, herramientas y población objeto de estudio se trabajó con la participación de 30 docentes de la Unidad Educativa Yelmo Rivadeneira Caicedo de Manabí - Ecuador en la aplicación de encuesta, una entrevista a un experto.

Para valorar las competencias lingüísticas se planteó la aplicación de técnicas creativas en sesiones de clase durante una semana con la participación activa de 10 estudiantes considerado como grupo focal, a través de una batería de preguntas se evaluaron las habilidades mediante rúbrica para el análisis de estudio. (Tabla 1) 
Técnica creativa de comprensión

lectora: Transformación de la narración.

Actividad principal:
- Cambiar los elementos de la historia de "María Panchita cocina con su abuela"

- Competencias a Evaluar: Leer

y escuchar.

Tabla 1. Competencias lingüísticas.

\begin{tabular}{|l|l|}
\hline \multicolumn{2}{|c|}{ COMPETENCIAS LINGÜÍSTICAS A OBSERVAR } \\
\hline \multicolumn{1}{|c|}{ Leer } & \multicolumn{1}{c|}{ Escuchar } \\
\hline $\begin{array}{l}\text { Reinventa textos literarios reconociendo la } \\
\text { fuente original, los relaciona con el texto }\end{array}$ & $\begin{array}{l}\text { Escucha discursos orales y formula juicios de } \\
\text { cultural, propio y de otros entornos. }\end{array}$ \\
& $\begin{array}{l}\text { de manespeto a sus contenidos y participa } \\
\text { intervenciones de los demás. }\end{array}$ \\
\hline
\end{tabular}

\begin{tabular}{|c|c|c|c|}
\hline \multirow[b]{2}{*}{$\begin{array}{l}\text { COMPENTENCIA } \\
\text { LINGÜÍSTICA A } \\
\text { EVALUAR: }\end{array}$} & \multicolumn{3}{|c|}{ VALORACIÓN } \\
\hline & $\begin{array}{l}\text { 1.- Competencia } \\
\text { Lograda: } \\
\text { Reinventa el texto } \\
\text { del cuento } \\
\text { respetando las } \\
\text { ideas centrales y de } \\
\text { forma coherente. }\end{array}$ & 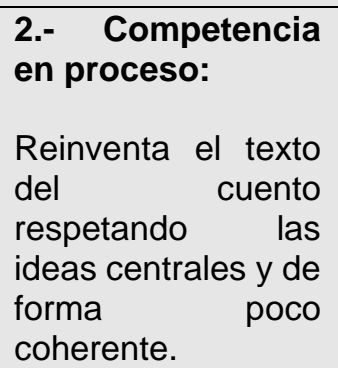 & $\begin{array}{l}\text { 3.- Competencia } \\
\text { no lograda: } \\
\text { Reinventa el texto } \\
\text { del cuento sin } \\
\text { respetar las ideas } \\
\text { centrales y sin } \\
\text { coherencia }\end{array}$ \\
\hline $\begin{array}{l}\text { Leer: Reinventa textos } \\
\text { literarios reconociendo } \\
\text { la fuente original, los } \\
\text { relaciona con el texto } \\
\text { cultural, propio y de } \\
\text { otros entornos. }\end{array}$ & & & \\
\hline $\begin{array}{l}\text { COMPENTENCIA } \\
\text { LINGÜÍSTICA A } \\
\text { EVALUAR: }\end{array}$ & $\begin{array}{l}\text { 1.- Competencia } \\
\text { lograda: } \\
\text { Muestra una } \\
\text { escucha atenta y } \\
\text { respetuosa durante } \\
\text { la narración de la } \\
\text { docente y la } \\
\text { participación de los } \\
\text { compañeros-as. }\end{array}$ & $\begin{array}{l}\text { 2. Competencia en } \\
\text { proceso: } \\
\text { Muestra una } \\
\text { escucha atenta y } \\
\text { respetuosa en } \\
\text { algunos momentos } \\
\text { de la narración de la } \\
\text { docente y la } \\
\text { participación de los } \\
\text { compañeros-as. }\end{array}$ & 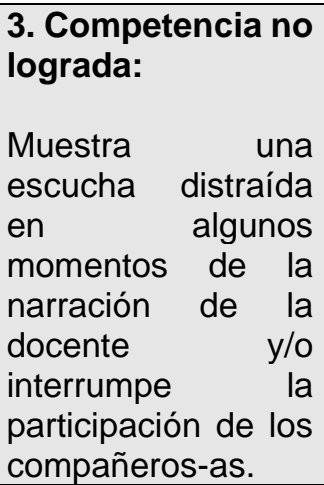 \\
\hline $\begin{array}{l}\text { Escuchar: Escucha } \\
\text { discursos orales y } \\
\text { formula juicios de valor } \\
\text { con respeto a sus } \\
\text { contenidos y participa } \\
\text { de manera respetuosa } \\
\text { frente a las } \\
\text { intervenciones de los } \\
\text { demás. }\end{array}$ & & & \\
\hline
\end{tabular}




\section{Resultados}

Los resultados demuestran que el $53 \%$ de docentes si aplican estrategias didácticas para mejorar el rendimiento de la comprensión lectora en los estudiantes de una manera probable. (Placencia, 2018) menciona que la población estudiantil ecuatoriana posee grandes dificultades para estructurar textos, existe baja calidad interpretativa y comprehensiva de los mismos. Se evidencia que la mayoría de niños de la educación general básica tienen una lectura silábica, problemas ortográficos y en su mayoría necesitan potenciar las competencias básicas de lenguaje: hablar, escuchar, leer y escribir.

Los datos indican que los docentes no están aplicando las técnicas de comprensión lectora, sin embargo, al no aplicarlas el estudiante, aunque domine el proceso de decodificación, conozca el vocabulario que la lectura contiene y esté motivado a leer, si al momento de hacerlo no utiliza las destrezas, será muy difícil que comprenda la lectura y adquiera conocimiento. Por ello es necesario ayudar a los estudiantes a utilizar técnicas lectoras de forma directa, sistemática y planificadas por los docentes en las instituciones educativas.

De acuerdo a los resultados obtenidos el $53 \%$ de docentes aplican la comprensión escrita muy frecuentemente en el desarrollo de sus temas de clases. (Tabla 2 encuesta docente). (Guaján, 2014) menciona que el desarrollo de destrezas de escuchar o leer es importante porque a través de ellas es que se comprende el habla oral. La comprensión auditiva y la expresión visual forman parte de la lengua que constituye un objetivo a largo plazo. Por eso quien es capaz de escuchar y pronunciar bien puede leer y escribir correctamente.

Los resultados indican que los docentes se esfuerzan sin éxito, en aplicar de manera adecuada las habilidades lingüísticas en el desarrollo de sus clases. Es menester dar a conocer a docentes que las competencias de escuchar y leer son habilidades receptivas mientras que hablar y escribir son habilidades productivas. Por tal razón los docentes deben aplicar las habilidades receptivas (escuchar y leer) se refieren a la comprensión través de la lectura y la escucha en 
sus clases para lograr desarrollar aquellas competencias productivas en los estudiantes. Enseñar a escuchar y leer puede ser muy diferente dependiendo de la metodología seguida por cada docente, pero lo que es realmente importante es desarrollar esta habilidad durante las clases.

Tabla 2. Con qué frecuencia aplican los docentes las competencias lingüísticas en el desarrollo de sus temas de clases.

MUY

ALTERNATIVAS FRECUENTE FRECUENTEMENTE OCACIONALMENTE RARAMENTE NUNCA TOTAL

\begin{tabular}{|c|c|c|c|c|c|}
\hline $\begin{array}{l}\text { Expresión } \\
\text { oral. }\end{array}$ & $47 \%$ & $43 \%$ & $10 \%$ & & $100 \%$ \\
\hline $\begin{array}{l}\text { Expresión } \\
\text { escrita }\end{array}$ & $47 \%$ & $43 \%$ & $10 \%$ & & $100 \%$ \\
\hline $\begin{array}{l}\text { Comprensión } \\
\text { lectora }\end{array}$ & $34 \%$ & $50 \%$ & $13 \%$ & $3 \%$ & $100 \%$ \\
\hline $\begin{array}{l}\text { Comprensión } \\
\text { escrita }\end{array}$ & $53 \%$ & $33 \%$ & $7 \%$ & $7 \%$ & $100 \%$ \\
\hline
\end{tabular}

El $50 \%$ de los docentes mencionaron que las técnicas creativas de la comprensión lectora más utilizada por docentes para desarrollar las competencias lingüísticas es leer un contenido (Tabla 3 encueta docente). Para (Boza, 2018, pág. 7) dan a conocer que las estrategias didácticas son muy buena influencia al momento de querer innovar y despertar la atención de los estudiantes, por eso, estas nuevas estrategias junto a la comprensión lectora harán que los estudiantes se vean motivados, desempeñándose a desarrollar y mejorar la comunicación dentro y fuera de las instituciones educativas.

Estos resultados muestran que los docentes han dejado de utilizar técnicas didácticas para el perfeccionamiento de la comprensión lectora en los estudiantes, los cuales estarían contentos con la aplicación de las técnicas de compresión lectora por sus docentes en las clases, con esto se estaría ayudando a optimizar en su rendimiento académico y las técnicas didácticas mejorarían la enseñanza hacia los educandos, forjando una educación más útil, de 
tal manera que impulse excelente desempeño en el transcurso académico de los estudiantes. Para ello es necesario ejercitarla y recibir una buena orientación en el aprendizaje. La comprensión lectora les servirá, indudablemente, como un método de desarrollo intelectual y emocional.

\section{Conclusiones}

Como resultado de la indagación sobre las técnicas creativas de la comprensión lectora factibles en el proceso de enseñanza virtual. Se comprobó que los docentes entrevistados emplean estrategias metodológicas tradicionales que evidencian la necesidad de un cambio en el enfoque de enseñanza y aprendizaje. Se plantea que el uso de las técnicas de comprensión lectora son herramientas didácticas útiles para la formación de lectores autónomos, las cuales ayudan al mejoramiento de la comprensión lectora; es decir, la implementación de las técnicas de comprensión lectora serán el fortalecimiento de la comprensión de lectura en los procesos dentro del aula.
Para establecer las formas de desarrollar las competencias lingüísticas en los estudiantes, se aplicó las siguientes técnicas creativas de compresión lectora, transformación de la narración, tramas narrativas, ficha de personajes, conversación escrita con un personaje, anuncio de una historia. Se comprueban que estas técnicas serán favorables para el desarrollo de las competencias lingüísticas en la etapa estudiantil como factibles en el proceso de enseñanza aprendizaje, las cuales serán facilitadoras de los procesos de comprensión lectora y, por tanto, deben ser enseñadas a los escolares desde sus primeros contactos con tareas que requieran comprensión de textos y por tanto implementadas por los docentes en sus clases debido que la mayoría de estudiantes no tiene hábitos de lectura ni alcanza los niveles de comprensión esperados.

Es importante reafirmar cómo el fortalecimiento de la acción pedagógica desde el mismo momento de la formación del docente se constituye en uno de los principales ejes que favorece la educación. Sin embargo, también se 
afirma la realidad de los profesores y

cómo estos deben cumplir con demasiadas actividades en su cotidianidad, lo cual en muchas ocasiones entorpece su correcto proceder con los estudiantes. Es necesario que las instituciones educativas brinden los espacios suficientes para que el maestro pueda planear y fortalecer su práctica docente enfocada en el aprendizaje y procesos de sus estudiantes.

Se requiere proponer la aplicación de técnicas creativas de comprensión lectora para mejorar la competencia lingüística de los estudiantes, por tal razón los docentes deben continuar capacitándose y formándose por medio de jornadas pedagógicas en lo que respecta a las dificultades en la comprensión lectora para buscar alternativas y soluciones a estas falencias. Este trabajo de investigación se proyecta como un documento de apoyo a otros profesores inquietos por buscar alternativas y fortalecer sus prácticas con el propósito de influir positivamente en los procesos cognitivos y personales de los estudiantes.

\section{Bibliografía}

Borragán, J. A. (2011). CÓMO ATRAER LA ATENCIÓN HABLANDO. UN RETO. Universidad de Cantabria; Bordón 63 (2), 20. Obtenido de

https://dialnet.unirioja.es/metr icas/documentos/ARTREV/36 57071

Boza, I. R. (2018). ESTRATEGIAS DIDÁCTICAS EN LA COMPRENSIÓN LECTORA. Guayaquil.: UNIVERSIDAD DE GUAYAQUIL. Obtenido de

http://repositorio.ug.edu.ec/ha ndle/redug/3538

Cervantes, D. C. (2017). Comprensión Lectora: Educación $Y$ Lenguaje. España: Palibrio. Obtenido de https://books.google.es/books $? \mathrm{id}=\_Y v E D g A A Q B A J \& l p g=P$ T3\&ots $=e Z w 1 E n 3 O g a \& d q=q$ ue $\% 20$ es $\% 20$ comprension\% 20lectora\&|r\&hl=es\&pg=PT3\# $\mathrm{v}=$ onepage $\& \mathrm{q}=$ Daniel $\% 20 \mathrm{Ca}$ nt\%C3\%BA\%20Cervantes\&f $=$ false

Chávez, M. (2015). Estrategias didácticas para mejorar la comprensión lectora. MÉXICO: www.monografias.com.

Obtenido de https://www.monografias.com /trabajos93/estrategiadidactica-mejoracomprension- 
lectora/estrategia-didacticamejora-comprensionlectora.shtml\#C

Cordero Badilla, M. N. (2018). El uso de técnicas de gamificación para estimular las competencias lingüísticas de estudiantes. Revistade Lenguas ModeRnas, N. ${ }^{\circ}$ 28, 2018, $277 . \quad$ doi: https://doi.org/10.15517/rlm.v 0i28.34777

Guaján. (2014). ESTUDIO DEL USO DE LA CREATIVIDAD EN EL DESARROLLO DE. Ibarra: UNIVERSIDAD TÉCNICA DEL NORTE.

Herrera, E. M. (2010). Factores que inciden en la comprensión lectora. ARGENTINA: UNIVERSIDAD CATOLICA DE CUYO.

Laguardia, K. G. (2016). COMPRENSIÓN LECTORA, MEMORIA DE TRABAJO, FLUIDEZ Y VOCABULARIO EN ESCOLARES CUBANOS. Revista Electrónica "Actualidades Investigativas en Educación", 4. doi: http://dx.doi.org/10.15517/aie. v16i1.21715

Ministerio de Educación del Ecuador. (2016). Ministerio de Educación. Obtenido de www.educacion.gob.ec

Miranda, P. (2015). ESTREATEGIA DE APRENDIZAJE, MOTIVACIÓN PARA EL
ESTUDIO Y COMPRENSIÓN LECTORA DE LOS ESTUDIANTES. PERÚ: UNIVERSIDAD NACIONAL MAYOR DE SAN MARCOS. Obtenido de https://hdl.handle.net/20.500. 12672/4322

Moren, A. E. (2018). La importancia de la emoción en el aprendizaje: Propuestas para mejorar la motivación de los estudiantes. Cuaderno de Pedagogía Universitaria Vol. $15 /$ no. 2, 6. Obtenido de https://dialnet.unirioja.es/busc ar/documentos?querysDisma $x . D O C U M E N T A L \quad T O D O=L a$ +importancia+de+la+emoci\% C3\%B3n+en+el+aprendizaje $\% 3 \mathrm{~A}+$ Propuestas+para+mejo rar+la+motivaci\%C3\%B3n+d e+los+estudiantes

Murillo, R. M. (2009). LA HABILIDAD DE ESCUCHAR. UNA TAREA PENDIENTE EN LA EDUCACIÓN. Revista Káñina, vol. XXXIII, 105.

Neyra, S. K. (2017). USO DE VIDEOS EDUCATIVOS COMO RECURSO DIDÁCTICO. Perù: Universidad San Martin de Porres. Obtenido de https://hdl.handle.net/20.500. 12727/3049

Ortega, V. (2017). ¿TE ANIMAS A LEER? UNA EXPERIENCIA DE ANIMACIÓN A. DIDACTICAE: REVISTA DE INVESTIGACION EN 
DIDACTICAS ESPECIFICAS ISSN 2462-2737, 131. doi: https://doi.org/10.1344/did.20 19.5.130-144

Pacheco, N. (2018). TÉCNICAS DE APRENDIZAJE LÚDICO PARA EL DESARROLLO DE COMPETENCIAS

LINGÜÍSTICAS. Guayaquil: Universidad de Guayaquil. Obtenido de http://repositorio.ug.edu.ec/ha ndle/redug/36034

Pérez., M. M. (2004). Aproximaciones conceptuales en torno al hablar y la competencia. Revista Varela, 4(6), 1-9., 3.

Plaza, Á. M. (2014). LA INFLUENCIA DE LA MEMORIA Y LAS ESTRATEGIAS DE APRENDIZAJE EN RELACIÓN A LA COMPRENSIÓN LECTORA EN ESTUDIANTES DE EDUCACIÓN PRIMARIA. INFAD Revista de Psicología, №1-Vol.6, 344.

Ruiz, D. R. (2019). ESCUCHAR Y LEER. UN ACERCAMIENTO AL DIAGNÓSTICO DE HABILIDADES LINGÜÍSTICAS

RECEPTIVAS EN ESTUDIANTES DE LA UNIVERSIDAD AUTÓNOMA. REVISTA INCLUSIONES ISSN 0719-4706 VOLUMEN 6, 140.
Salazar, D. J. (2018). ESTRATEGIAS DIDÁCTICAS EN LA COMPRENSIÓN LECTORA.GUIA. GUAYAQUIL: UNIVERSIDAD DE GUAYAQUIL. Obtenido de http://repositorio.ug.edu.ec/ha ndle/redug/35623 